\title{
The continuity of prime numbers can lead to even continuity(Goldbach conjecture)
}

\section{Xie Ling ( 29997609@qq.com )}

Cosmic Mathematical Physics

\section{Research Article}

Keywords: prime even continuity, Bertrand Chebyshev theorem, Ascending and descending, Extreme law, Mathematical complete induction

Posted Date: November 16th, 2021

DOI: https://doi.org/10.21203/rs.3.rs-713902/v18

License: (9) This work is licensed under a Creative Commons Attribution 4.0 International License. Read Full License 


\title{
The continuity of prime numbers can lead to even continuity(Goldbach conjecture)
}

\author{
Author: Xie Ling \\ Address: College of cosmic mathematics and physics, China / Hunan \\ E-mail: 29997609@qq.com Mobile phone: 13973920402 \\ Mathematical classification (MSC2020):11P32;11U05:11N05:11P70 \\ PREPRINT(UNPUBLISHED VERSION)ＤOI:10.21203/rs.3.rs-713902/v17
}

\begin{abstract}
Research ideas:
If the prime number is continuous, and any pairwise addition can obtain even number continuity, then Goldbach's conjecture is true.

Human even number experiments all get (prime number + prime number).

I propose a new topic: the continuity of prime numbers can lead to even continuity.

I designed a continuous combination of prime numbers and got even continuity.

If the prime numbers are combined continuously and the even numbers are forced to be discontinuous, a breakpoint occurs.

It violates Bertrand Chebyshev's theorem.

It is proved that prime numbers are continuous and even numbers are continuous.

The logic is: if Goldbach conjecture holds, it must be that the continuity of prime numbers can lead to the continuity of even numbers.
\end{abstract}

Image interpretation: turn Goldbach's conjecture into a ball, and I kick the ball into Goldbach's conjecture channel.

There are several paths in this channel, and the ball is not allowed to meet Goldbach's conjecture conclusion in each path.

This makes the ball crazy, and the crazy ball must violate Bertrand Chebyshev's theorem. 
Absrtact : Two prime numbers $\{3,5\}$.

$\{3,5\} \rightarrow\{3+3=6,3+5=8,5+5=10\} \rightarrow\{6,8,10\}$.

$\{\{10\} \rightarrow(5+5=10=3+7) \rightarrow 7\}$ Increased by $7 \rightarrow\{3,5,7\}$.

Note changes: $\{3,5\} \rightarrow\{6,8,10\} \rightarrow\{3,5,7\}$.

$\{3,5,7\} \rightarrow\{3+3=6,3+5=8,5+5=10,5+7=12,7+7=14\} \rightarrow\{6,8,10,12,14\}$.

$\{\{14\} \rightarrow(7+7=14=3+11) \rightarrow 11\}$ Increased by $11 \rightarrow\{3,5,7,11\}$.

Note changes: $\{3,5,7\} \rightarrow\{6,8,10,12,14\} \rightarrow\{3,5,7,11\}$.

The same logic would be: $\{3,5,7,11\} \rightarrow\{6,8,10,12,14,16\} \rightarrow\{3,5,7,11,13\}$.

The same logic would be:

$\{3,5,7,11,13\} \rightarrow\{6,8,10,12,14,16,18,20\} \rightarrow\{3,5,7,11,13,17\}$.

【Note: if a prime number is added, $n$ consecutive even numbers $(n \geqslant 1)$ can be obtained.】

Sequential continuation,

If you expand infinitely in the above specified mode: $\{3,5,7,11,13,17,19,23, \ldots$.

Get: $\{6,8,10,12,14,16,18,20,22, \ldots$

The above is: the continuity of prime numbers can lead to even continuity.

Get: Goldbach conjecture holds.

If it is mandatory: Authenticity stops at an even number $2 \mathrm{n}$.

$\{\{3,5,7,11,13,17,19,23, \ldots ., p 1\} \rightarrow\{6,8,10,12,14,16,18,20,22, \ldots, 2 n\}$.

$\left\{3,5,7,11,13,17,19,23, \ldots, p_{1}\right\} \nrightarrow(2 \mathrm{n}+2)$.

$\forall p+\forall p \neq \mathbf{2 n}+\mathbf{2}\}$ It can be proved that: It violates the "Bertrand Chebyshev" theorem.

$\therefore\{3,5,7,11,13,17,19,23, \ldots$

$\rightarrow\{6,8,10,12,14,16,18,20,22, \ldots$

Get: Goldbach conjecture holds.

Key words: prime even continuity; Bertrand Chebyshev theorem; Ascending and descending; Extreme law; Mathematical complete induction

Background of the study

Goldbach conjecture ${ }^{[1]}$ : Euler's version, that is, any even number greater than 2 can be written as the sum of two prime numbers, also known as "strong Goldbach conjecture" or "Goldbach conjecture about even numbers".

It was put forward in 1742, and today it has trapped excellent mathematicians of mankind.

Is this Goldbach conjecture correct? Or wrong?

Why can't humans find a way to prove or falsify?

It is because human beings do not find the right path by definition, and human beings have 
gone astray.

Purpose of the study

Starting with the definition, find out the internal relationship between Goldbach conjecture and mathematical logic,

Confirm Goldbach conjecture or deny Goldbach conjecture.

Prove the distribution law of prime numbers.

The summary of the existing literature proves that the study of Goldbach conjecture is correct.

\section{1}

\section{Text: introduction}

1.1 Research skills: The whole proof begins with the ascent

1.1.1 Even number generation provisions in this paper

Rule (1): the minimum odd prime number is $\mathbf{3}$

Rule (2): add two odd prime numbers.(any combination of two odd primes).

Rule (3): odd prime number can be quoted repeatedly.

Rule (4):meet the previous provisions, and all prime combination to the maximum(for example, $10=5+5$ must be: $10=5+5=3+7$,

For another example, the combination of 90 must be: $90=43+47=37+53=31+$ $59=29+61=23+67=19+71=17+73=11+79=7+83)$.

Rule (5): $\mathrm{P}_{\mathrm{a}}+\mathrm{P}_{\mathrm{b}}=2 \mathrm{~S}$, and $\mathrm{P}_{\mathrm{b}}+\mathrm{P}_{\mathrm{a}}=2 \mathrm{~S}$, Delete one and leave only one.

\subsubsection{I can quote the minimum odd prime number $\mathbf{3}$}

The results are as follows

$3+3=6$

$\mathbf{5}+\mathbf{1}=\mathbf{6}$ (it is stipulated that $\mathbf{1}$ is not a prime number, which is deleted because it violates regulation 2 )

$\therefore$ The unique formula: $3+3=6$.(comply with Rule (4): all prime numbers are quoted to the maximum).

$3 \rightarrow 6$

1.1.3 Even number generation provisions in this paper: Get the following

$\{3\} \rightarrow(3+3)=6$

$\{3,5\} \rightarrow(3+5)=8$

$\{3,5\} \rightarrow(5+5)=10 \rightarrow(3+7) \rightarrow\{7\} \quad \therefore\{3,5,7\}$

$\{3,5,7\} \rightarrow(5+7)=12$

$\{3,5,7\} \rightarrow(7+7)=14 \rightarrow(3+11) \rightarrow\{11\} \quad \therefore\{3,5,7,11\}$

$\{3,5,7,11\} \rightarrow(11+5)=16 \rightarrow(3+13) \rightarrow\{13\} \quad \therefore\{3,5,7,11,13\}$ 
Look: prime continuous $3 \rightarrow 5 \rightarrow 7 \rightarrow 11 \rightarrow 13$

Look: you get "even numbers are also continuous" $6 \rightarrow 8 \rightarrow 10 \rightarrow 12 \rightarrow 14 \rightarrow 16$

If you let the prime number be infinitely continuous: $3 \rightarrow 5 \rightarrow 7 \rightarrow 11 \rightarrow 13 \rightarrow 17 \rightarrow \ldots$

"Even numbers are also infinitely continuous": $6 \rightarrow 8 \rightarrow 10 \rightarrow 12 \rightarrow 14 \rightarrow 16 \rightarrow 18 \rightarrow \ldots$

A new theorem is obtained: The continuity of prime numbers can lead to even continuity。

If this theorem is proved, Goldbach's conjecture is correct.

1.2 The proof process uses the known prime number,

Continuity of prime numbers:

$\because$ The theorem of infinite number of primes $^{[3]}$ :

(1) There is no maximum prime;

(2)After each prime, you can always find an adjacent prime.

$\therefore$ Prime numbers have continuity.

Prime number theorem ${ }^{[3]}$ and Bertrand Chebyshev ${ }^{[2]}$ theorem.

By quoting the prime theorem (Each prime has a subsequent adjacent prime.), we obtain the unrestricted continuity of prime numbers, and then generate even numbers according to the requirements of this paper, If the generated even number is infinitely continuous, Goldbach's conjecture is correct.

Use the limit rule to force the even number to terminate at $2 n=($ Prime $)+($ prime $)$, and the even number $2 n+2=($ Prime $)+($ prime $)$ cannot occur.

Conclusion:

If it conforms to mathematical logic, then: Goldbach conjecture is wrong.

If it does not conform to mathematical logic, then Goldbach's conjecture is correct.

\section{Text: nouns and definitions}

2.1 $\{$ Definition of prime number: prime number refers to the natural number with no other factors except 1 and itself in the natural number greater than 1.\}record: (2.1)

2.2 Extreme law : A may or may not be true. What conclusion can we get if we only prove that $\mathbf{A}$ is not true. 
$\{\mathbf{A} \mid \mathbf{A}=\mathbf{x}, \mathbf{A}=\mathbf{y}\},(\mathbf{A}=\mathbf{x}) \Rightarrow(Q E D)$. Take: $\mathbf{A} \neq \mathbf{x}$, only prove the $\mathbf{A}=\mathbf{y}$ conclusion. $\}$ record: $(2.2)$

2.3 \{ [References cited $\left.{ }^{[2]}\right]$ Bertrand Chebyshev theorem: if the integer $\mathbf{n}>\mathbf{3}$, then there is at least one prime $\mathbf{P}$, which conforms to $\mathbf{n}<\mathbf{p}<\mathbf{2 n - 2}$. Another slightly weaker argument is: for all integers $n$ greater than $\mathbf{1}$, there is at least one prime $\mathrm{P}$, which conforms to $\mathbf{n}<\mathbf{p}<\mathbf{2 n}$. $\}$ record:: (2.3)

2.4 $\{$ The generation of even and prime numbers in this paper is specified as follows:

In this paper, the even number generation rules: the following five rules are met at the same time.

(1) Only odd primes are allowed as elements.

(2) Only two prime numbers can be added.(any combination of two odd primes).

(3) Two prime numbers can be used repeatedly: $(\mathbf{3}+\mathbf{3})$, or $(\mathbf{3}+\mathbf{5})$, or $(\mathbf{P}+\mathbf{P})$.

(4) meet the previous provisions, and all prime combination to the maximum(for example, $10=5+5$ must be: $10=5+5=3+7$,

For another example, the combination of 90 must be: $90=43+47=37+53=31+$ $59=29+61=23+67=19+71=17+73=11+79=7+83)$.

(5) Take only one of $\left(\mathbf{p}_{\mathbf{a}}+\mathbf{p} \mathbf{b}\right) \operatorname{and}\left(\mathbf{p}_{\mathbf{b}}+\mathbf{p}_{\mathbf{a}}\right)$.

In this paper, the generation rules of prime numbers: the following three rules are met at the same time.

(1) The first odd prime number is 3 .

(2) Get the prime number from the even number. For example, $3+5=8$. Prime numbers 3 and 5 are the materials I can cite.

(3) Get the prime number from the even number. It must be two prime numbers. For example, $3+5=8$. In the combination of even number 8 , the prime numbers 3 and 5 are the materials I can cite.

For example, $1+7=8$. In the combination of even number 8 , I cannot quote prime number 7.$\}$ record: (2.4)

A pseudo stop property is obtained in (2.4):

2.41 \{Pseudo stop: Explain first. the first prime number 3, according to the even number generation regulations, gets $3+3=6$. Even number 6 , according to the prime generation regulations, cannot generate 5 , because $(1+5)$ does not meet the even number 6 in this paper, 
cannot generate quality 5 . So there is a false stop: only 3 , no 5 , only 6 , no 8 . But you can artificially increase prime number 5 , and you can also get $8(3+5)$. So it is a false stop.

Pseudo stop definition:

(1) the maximum even number among the continuous even numbers composed of $\mathrm{k}$ consecutive prime numbers $\left\{3,5,7,11, \ldots, \mathrm{P}_{1}\right\}$ is $2 \mathrm{n}$

(2) $\left\{3,5,7,11, \ldots, \mathrm{P}_{1}\right\}$ can meet: $\{6,8,10,12,14,16,18,20, \ldots, 2 \mathrm{n}\}$

(3) $\left\{3,5,7,11, \ldots, P_{1}\right\}$ not satisfied: $\{2 n+2\}$

(4) Odd prime $\mathrm{P}_{0}: \mathrm{P}_{0} \notin\left\{3,5,7,11, \cdots, \mathrm{P}_{1}\right\}$, satisfying: $\left.2 \mathrm{n}+2=\mathrm{P}_{0}+\mathrm{P}_{\mathrm{y}}\right\}$ record: (2.41)

2.42 \{rue stop: two prime numbers $\mathrm{P}_{1}$ and $\mathrm{P}_{2}$, satisfying: $\mathrm{P}_{1}+\mathrm{P}_{2}=2 \mathrm{n}$. Any two prime numbers $\mathrm{P}_{\mathrm{X}}$ and $\mathrm{P}_{\mathrm{y}}$ cannot satisfy: $\left.\mathrm{P}_{\mathrm{X}}+\mathrm{P}_{\mathrm{y}}=2 \mathrm{n}+2.\right\}$ record: (2.42)

2.5 \{ [References cited $\left.{ }^{[3]}\right] \quad$ The theorem of infinite number of primes:

the $\mathrm{n}$ bit after each prime can always find another prime.

For example, $\mathbf{3}$ is followed by $\mathbf{5}$ and $\mathbf{1 3}$ is followed by 17; There must be an adjacent prime $\mathbf{p}_{\mathbf{i}}$ after the prime $\mathbf{P}$. $\}$ record: (2.5)

2.6 $\{$ Here we only discuss the following cases: prime number sequence and even number sequence

$((2.5)) \Rightarrow$ Prime number sequence: $\mathbf{3}, \mathbf{5}, \mathbf{7}, \mathbf{1 1}, \mathbf{1 3}, \mathbf{1 7}, \mathbf{1 9}, \mathbf{2 3}, \ldots$

Even number sequence: $6,8,10,12,14,16,18,20, \ldots$

Explain (2.5) in today's words: the prime number in the prime number sequence discussed in this paper is adjacent and continuous, and the first number is $\mathbf{3}$.

An even number in an even number sequence is contiguous and the first number is $\mathbf{6}$. record: $(2.6)$

2.7 $\{$ Remember: all the primes I'll talk about below refer to odd primes (excluding 2).

Prime symbol $\mathbf{P}$, different primes use $\left.\mathbf{P}_{\mathbf{1}}, \mathbf{P}_{\mathbf{2}}, \mathbf{P}_{\mathbf{3}}, \ldots, \mathbf{P}_{\mathbf{x}}\right\}$ record: (2.7)

3 Text: Logical discussion (argument) and results

3.1 $\left\{\right.$ Goldbach conjecture ${ }^{[1]}: 3 \leqslant \forall n \in N, \quad 2 n=\mathbf{p}_{x}+\mathbf{p}_{\mathrm{y}} \cdot$ set up $\left.\Delta_{1}: \mathbf{p}_{\mathbf{x}} \geqslant \mathbf{p}_{\mathrm{y}}\right\}$ 
record: (3.1)

3.2 Theorem: the continuity of prime numbers leads to the continuity of even numbers.

In mathematical language :

Known: $\left\{\mathbf{3}, \mathbf{5}, \mathbf{7}, \mathbf{1 1}, \mathbf{1 3}, \ldots, \mathbf{\mathbf { p } _ { 2 }}, \mathbf{p}_{1}, \mathbf{p}_{\mathbf{0}}\right\} \in($ prime$)$, The next neighbor of $\mathbf{p}_{1}$ is po,

$3<5<7<11<13<\ldots p_{2}<p_{1}<p_{0}$,

$\{6,8,10,12,14,16, \ldots . ., 2 \mathrm{n}\} \in$ (continuous even number).

If : $\left\{3,5,7,11,13, \ldots, p_{2}, p_{1}\right\} \Rightarrow\{6,8,10,12,14,16, \ldots, 2 n\}$.

inevitable: $\left\{3,5,7,11,13, \ldots, p_{2}, p_{1}, p_{0}\right\} \Rightarrow\{6,8,10,12,14,16, \ldots$,

$\mathbf{2 n}, \mathbf{2}(\mathbf{n}+\mathbf{1})\}$.$\} record: (3.2)$

\subsection{1 prove:}

Humans use computers to calculate a finite number of even numbers: $\{\mathbf{6 , ~ 8 , ~ 1 0 , 1 2 , ~}$ $14, . . ., 2 \mathrm{n}\}$ every even number satisfies (3.1).

The computer process is finite $\{\mathbf{6}, \mathbf{8}, \mathbf{1 0}, \mathbf{1 2}, \mathbf{1 4}, \ldots, \mathbf{2 n}\}$

It is not logically proved that any even number greater than $\mathbf{4}$ satisfies (3.1).

$((2.5)+(2.6))$ Take odd prime sequence: $\mathbf{3}, \mathbf{5}, \mathbf{7}, \mathbf{1 1}, \mathbf{1 3}, \mathbf{1 7}, \mathbf{1 9}, \mathbf{2 3}, \ldots$

Take the minimum prime number 3 from the front of the prime sequence,

A1: $\{(2.1)+(2.4)+\{3\}\} \Rightarrow$

$\{3+3=6\} \quad$ It is recorded as $\mathbf{A}_{1}$

$\rightarrow 6$

According to the rule, 3 can only get $\{3+3=6\}$

Nonexistence: $\mathbf{5}+\mathbf{p}=\mathbf{6}$

【Because $\mathbf{1}$ in $\mathbf{5 + 1}=\mathbf{6}$ is not defined as a prime number. If $\mathbf{1}$ is defined as a prime number, this paper will come to the same conclusion】

Note: $\mathbf{5} \notin\{\mathbf{3}\}$.

Prime number 3 , limit is used according to (2.4), cannot be: $\mathbf{6} \rightarrow \mathbf{8}$.

$\{(2.1)+(2.4)+\{3\}\} \Rightarrow \quad$ : Quoting prime number 3 can only get even number 6 .

A pseudo stop occurred (2.41).

If you want to: $\mathbf{6} \rightarrow \mathbf{8}$, you must add an adjacent prime number 5 .

$\therefore\{3,5\}$

$\Rightarrow 3 \rightarrow 5$ 
A2: $\{(2.1)+(2.4)+\{3,5\}\} \Rightarrow$

$3+3=6$

$3+5=8$

$\mathbf{5}+\mathbf{5}=\mathbf{3}+7=\mathbf{1 0} \quad \because(2.4) \Rightarrow\{(\mathbf{5}+\mathbf{5}),(\mathbf{3}+7)\} \in \mathbf{1 0} . \quad \therefore\{(2.1)+(2.4)+\{3,5,7\}\} \Rightarrow$

$7+5=12$

$7+7=11+3=14 . \quad \because(2.4) \Rightarrow\{(7+7),(3+11)\} \in 14 . \quad \therefore\{(2.1)+(2.4)+\{3,5,7,11\}\} \Rightarrow$

$11+5=13+3=16 \because(2.4) \Rightarrow\{(11+5),(13+3)\} \in 16 . \quad \therefore\{(2.1)+(2.4)+\{3,5,7,11,13\}\} \Rightarrow$

$11+7=13+5=18$.

$\mathbf{1 3}+\mathbf{7}=\mathbf{1 7}+\mathbf{3}=\mathbf{2 0}$. There is a new prime number 17 in continuity.

Note: $\{(13+7),(17+3)\} \in 20$. even numbers $\Rightarrow: 6,8,10,12,14,16,18,20$.

Prime numbers are continuous, and there is a new prime number 17.

$3 \rightarrow 5 \rightarrow 7 \rightarrow 11 \rightarrow 13 \rightarrow 17$.

even numbers $\Rightarrow: 6 \rightarrow 8 \rightarrow 10 \rightarrow 12 \rightarrow 14 \rightarrow 16 \rightarrow 18 \rightarrow 20$.

Wonderful continuity:

$\{\{\{3,5\} \rightarrow\{6,8,10\}$.

$(10) \rightarrow(10=3+7) \rightarrow 7\} \rightarrow\{3,5,7\}$.

$\{\{3,5,7\} \rightarrow\{6,8,10,12,14\}$.

$(14) \rightarrow(14=3+11) \rightarrow 11\} \rightarrow\{3,5,7,11\}$.

$\{\{3,5,7,11\} \rightarrow\{6,8,10,12,14,16\}$.

$(16) \rightarrow(16=3+13) \rightarrow 13\} \rightarrow\{3,5,7,11,13\}$.

$\{\{3,5,7,11,13\} \rightarrow\{6,8,10,12,14,16,18,20\}$.

$(20) \rightarrow(20=3+17) \rightarrow 17\} \rightarrow\{3,5,7,11,13,17\}$.

$\{\{3,5,7,11,13,17\} \rightarrow\{6,8,10,12,14,16,18,20,22\}$.

$(22) \rightarrow(22=3+19) \rightarrow 19\} \rightarrow\{3,5,7,11,13,17,19\}$.

$\{\{3,5,7,11,13,17,19\} \rightarrow\{6,8,10,12,14,16,18,20,22,24,26\}$.

$(26) \rightarrow(26=3+23) \rightarrow 23\} \rightarrow\{3,5,7,11,13,17,19,23\}\}$. Wonderful continuity is obtained: prime continuity, resulting in a new even continuity, and a new even number results in a new prime continuity. 
According to the rule (2.4) and the existing computer capabilities, the prime number 23 should be continuous to the subsequent prime number,

According to the rule (2.4) and the existing computer capacity, the even number 26 should be continuous to the following even number,

$\mathrm{A}_{2}$ has not stopped at this time.

Note the key point:

$\mathrm{A}_{1}$ pseudo stop, increase the adjacent prime number 5 to have $\mathrm{A}_{2}$.

From $A_{1} \rightarrow A_{2} \rightarrow$ is it always infinite? Or will it stop?

Here's the wonderful thing:

(analysis I ) :

Always Unlimited: $\quad \mathrm{A}_{1} \rightarrow \mathrm{A}_{2} \rightarrow \quad \ldots$

There are: $((2.5)+(2.6) \Rightarrow\{3,5,7,11, \mathbf{1 3}, \mathbf{1 7}, \mathbf{1 9}, \mathbf{2 3}, \ldots$

Get: $6,8,10,12,14,16,20,22, \ldots$

Conclusion : (3.2) (QED).

(2.2) $\Rightarrow \quad$ Stop at $A_{n}$, cannot continue.

(analysis II ): Stop at $A_{n}$, not to be continued.

Stop at $A_{n}: \quad A_{1} \rightarrow A_{2} \rightarrow \quad \ldots \rightarrow A_{n}$

$\kappa$-line with continuous Prime: $\mathbf{3}, \mathbf{5}, \mathbf{7}, \mathbf{1 1}, \mathbf{1 3}, \mathbf{1 7}, \mathbf{1 9}, \mathbf{2 3}, \ldots, \mathbf{p}_{2}, \mathbf{p}_{1}$

Get: $6,8,10,12,14,16,20,22, \ldots . ., 2(n-1), 2 n$

Terminate at $\mathrm{A}_{\mathrm{n}}$ to obtain: :

$$
\begin{gathered}
\left\{\left\{\left\{(2.1)+(2.4)+\left\{3,5,7,11, \ldots, p_{2}, p_{1}\right\}=\triangleright\{6,8, \mathbf{1 0}, \mathbf{1 2}, \ldots, \mathbf{2}(\mathbf{n}-\mathbf{1}), \mathbf{2 n}\}\right.\right.\right. \\
\left.\left.\quad \triangleright \quad\{\mathbf{6}, \mathbf{8}, \mathbf{1 0}, \mathbf{1 2}, \ldots, \mathbf{2 ( n - 1 )}, \mathbf{2 n}\} \in \mathrm{A}_{\mathrm{n}}\right\}\right\} \text { record: }(3.3)
\end{gathered}
$$

Terminate at $A_{n}$ to obtain: :

$\left\{\left\{\left\{(2.1)+(2.4)+\left\{3,5,7,11, \ldots, \mathbf{p}_{2}, \mathbf{p}_{1}\right\} \neq \triangleright \mathbf{2 ( n + 1 )} \Rightarrow\{\mathbf{2}(\mathbf{n}+\mathbf{1})\} \notin \mathrm{A}_{\mathrm{n}}\right\}\right\}\right.$ record: (3.4)

$\left\{\right.$ Let: prime p satisfy: $\mathbf{p} \notin\left\{3,5,7,11, \ldots, \mathbf{p}_{2}, \mathbf{p}_{1}\right\},(3.3),(3.4)$.

$\left.\therefore\left\{p_{1}<p,(p+\forall p) \notin\{6,8,10,12, \ldots, 2(n-1), 2 n\},(p+\forall p) \notin A_{n}\right\}\right\}$ 
Take the prime number that is greater than $\mathrm{p}_{1}$ and adjacent to $\mathrm{p}_{1}$ as $\mathrm{p}_{0}$,

$$
\begin{aligned}
& \because(1) \Rightarrow\left\{p_{0}+3 \neq 2 n \quad, p_{0}+3 \nless 2 n\right\} \\
& \therefore \quad p_{0}+3>2 n \\
& \therefore \quad p_{0}+2 \geqslant 2 n \quad \because \quad(\text { odd }) \neq(\text { even }) \\
& \therefore \quad p_{0}+2>2 n \quad \Rightarrow p_{0}+1 \geqslant 2 n \quad \operatorname{Record} \text { as }(W)
\end{aligned}
$$

\section{Starting from (Analysis II)}

The principle of mathematical complete induction:

it is correct in the front, until $\mathrm{A}_{\mathrm{n}}$.

Take the continuous prime number $\left(\mathbf{3 , 5}, \mathbf{7 , 1 1}, \ldots, \mathbf{p}_{2}, \mathbf{p}_{\mathbf{1}}\right)$ from small to large.

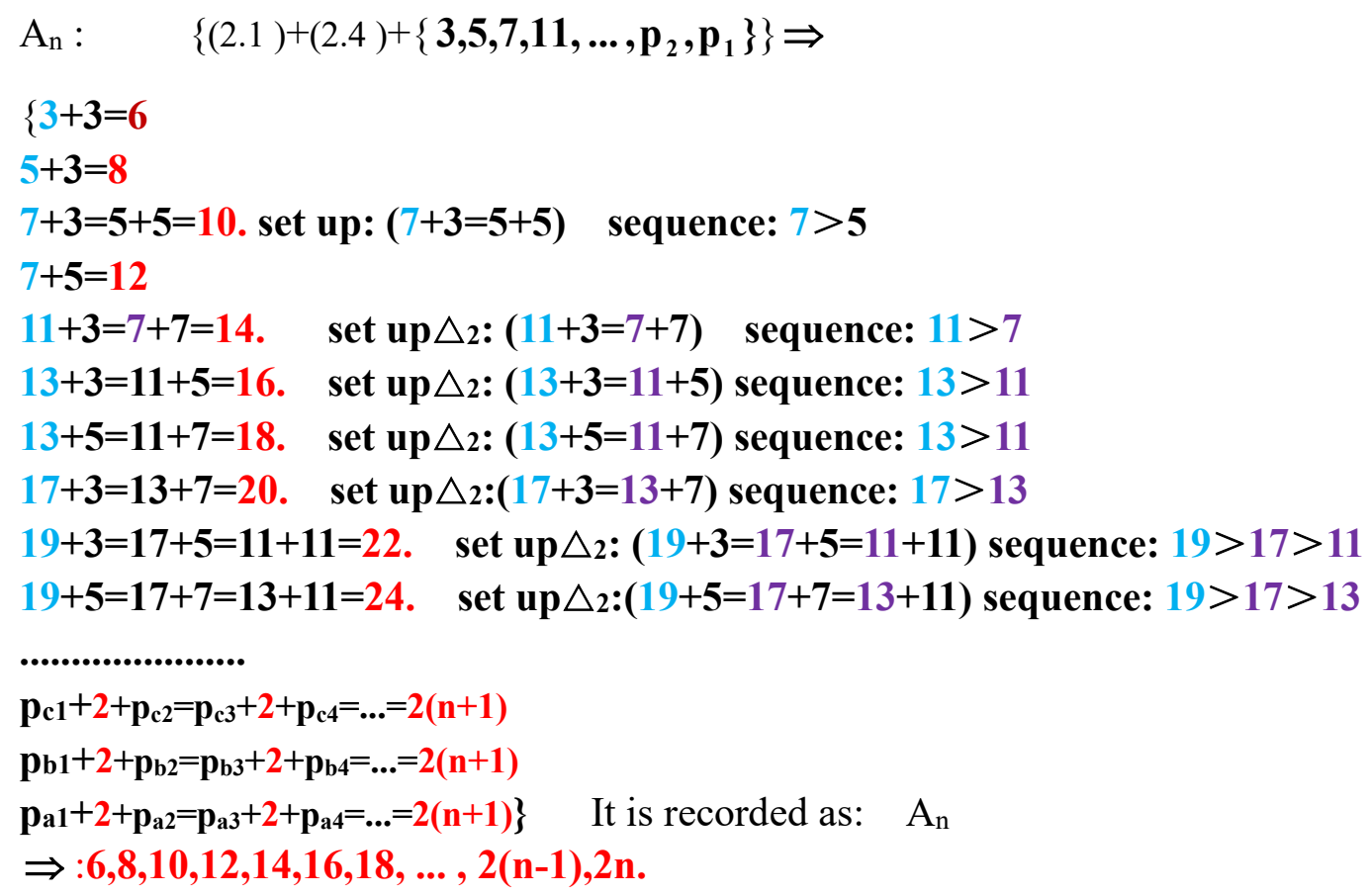

In $A_{n}$, it is specified that : $\mathbf{p}_{\mathbf{a} 1} \geqslant \mathbf{p}_{\mathbf{a} 2}$

In $A_{n}$ it is specified that $: \mathbf{p}_{\mathbf{a} 1}>\mathbf{p}_{\mathbf{a} 3}$ 【Reason: $\mathbf{p}_{\mathbf{a} 1}+\mathbf{p}_{\mathbf{a} 2}=\mathbf{2 n}$ must exist.

$\mathbf{p}_{\mathbf{a} 3}{ }^{+} \mathbf{p}_{\mathbf{a} 4}=\mathbf{2 n}$, not necessarily. If $\mathbf{p}_{\mathbf{a} 3}+\mathbf{p}_{\mathbf{a} 4}=\mathbf{2 n}$, exists $\mathbf{p}_{\mathbf{a} 1}$ and $\mathbf{p}_{\mathbf{a} 3}$ if one of them is big, put the big one in the first place according to the regulations.

If: $p_{a 1}=p_{a 3} \Rightarrow\left(p_{a 3}+p_{a 4}=2 n\right) \cong\left(p_{a 3}+p_{a 4}=2 n\right)$

$(2.4) \Rightarrow \mathbf{p a}_{\mathbf{a} 3}+\mathbf{p}_{\mathbf{a} 4}=\mathbf{2 n}$ is deleted $\quad \therefore \mathbf{p}_{\mathbf{a} 1}>\mathbf{p}_{\mathbf{a} 3}$ 
$\mathrm{A}_{\mathrm{n}}$ is simplified as $\mathrm{B}_{\mathrm{n}}$.

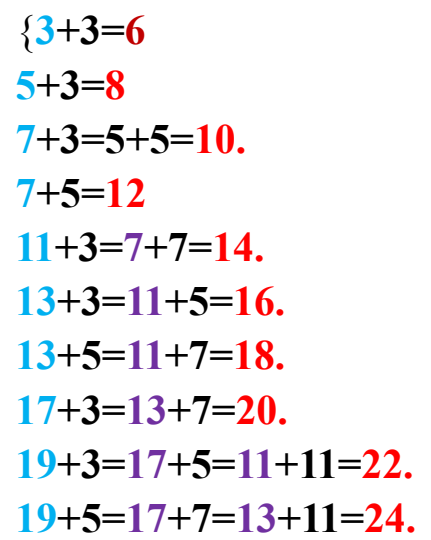

$\mathrm{p}_{\mathrm{c} 1}+2+\mathrm{p}_{\mathrm{c} 2}=\mathrm{p}_{\mathrm{c} 3}+2+\mathrm{p}_{\mathrm{c} 4}=\ldots=2(\mathrm{n}+1)$

$p_{b 1}+2+p_{b 2}=p_{b 3}+2+p_{b 4}=\ldots=2(n+1)$

$\left.\left.\mathbf{p}_{\mathrm{a} 1}+2+\mathbf{p}_{\mathrm{a} 2}=\mathbf{p}_{\mathrm{a} 3}+2+\mathbf{p}_{\mathrm{a} 4}=\ldots=\mathbf{n}+\mathbf{n}\right)\right\} \quad$ It is recorded as: $\mathrm{B}_{\mathrm{n}}$

Change $B_{n}$ to $C_{n}$.

$\{$ Level 2(n-2):3+2(n-2)+3=2(n+1)

Level 2(n-3): $5+2(n-3)+3=2(n+1)$

Level 2(n-4): $7+2(n-4)+3=5+2(n-4)+5=2(n+1)$

Level 2(n-5): $7+2(n-5)+5=2(n+1)$

Level 2(n-6): $11+2(n-6)+3=7+2(n-6)+7=2(n+1)$

Level 2(n-7): $13+2(n-7)+3=11+2(n-7)+5=2(n+1)$

Level 2(n-8): $13+2(n-8)+5=11+2(n-8)+7=2(n+1)$

Level 2(n-9): $17+2(n-9)+3=13+2(n-9)+7=2(n+1)$

Level 2(n-10): 19+2(n-10) $+3=17+2(n-10)+5=11+2(n-10)+11=2(n+1)$

Level $2(n-11): 19+2(n-11)+5=17+2(n-11)+7=13+2(n-11)+11=2(n+1)$

Level 6:p $p_{\mathrm{c} 1}+2+\mathrm{p}_{\mathrm{c} 2}=\mathrm{p}_{\mathrm{c} 3}+2+\mathrm{p}_{\mathrm{c} 4}=\ldots=2(\mathrm{n}+1)$

Level 4:p $p_{b 1}+2+p_{b 2}=p_{b 3}+2+p_{b 4}=\ldots=2(n+1)$

Level 2: $\left.\mathbf{p}_{\mathbf{a} 1}+2+\mathbf{p}_{\mathbf{a} 2}=\mathbf{p}_{\mathbf{a} 3}+2+\mathbf{p}_{\mathbf{a} 4}=\ldots=\mathbf{2}(\mathrm{n}+1)\right\} \quad$ It is recorded as : $\mathrm{C}_{\mathrm{n}}$

【friendly note: $\mathbf{C}_{\mathbf{n}}$ is Goldbach's conjecture channel. The branches of this channel are: Level 2 , level 4, level 6,... Level 2 (n-3), level 2 (n-2)】

"Line $\beta \quad$ " in $C_{n}, 2 \rightarrow 4 \rightarrow 6 \rightarrow 8 \rightarrow \ldots \rightarrow 2(n-5) \rightarrow 2(n-4) \rightarrow 2(n-3) \rightarrow 2(n-2)$

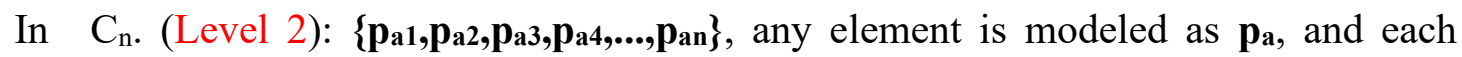
prime is abbreviated as $\mathbf{p}_{\mathbf{a}}$.

In $\mathrm{C}_{\mathrm{n}}$. (Level 4): $\left\{\mathbf{p}_{\mathbf{b} 1}, \mathbf{p}_{\mathbf{b} 2}, \mathbf{p}_{\mathbf{b} 3}, \mathbf{p}_{\mathbf{b} 4}, \ldots, ., \mathbf{p}_{\mathbf{b n}}\right\}$, any element is modeled as $\mathbf{p}_{\mathbf{b}}$, and each prime is abbreviated as $\mathbf{p}_{\mathbf{b}}$,

The same analogy follows (at each level).

Important note: in $C_{n}$, I didn't specify $\mathbf{p}_{\mathbf{a} 1}>\mathbf{p}_{\mathbf{b} 1}$, I didn't specify their size 
relationship.

\subsubsection{Theorem $\left(\omega_{1}\right)$ :}

$\left\{\mathrm{p}_{1}\right.$ and $\mathrm{p}_{0}$ are adjacent prime numbers. $\left.\quad \mathrm{p}_{0}>\mathrm{p}_{1}\right\} \Rightarrow \mathrm{p}_{0} \ngtr 2 \mathrm{p}_{1}$.

\section{Proof:}

Assumptions: $\mathrm{p}_{0}>\mathbf{2} \mathrm{p}_{1}$.

$\Rightarrow: \mathrm{p}_{0}>2 \mathrm{p}_{1}>\mathrm{p}_{1}$.

$(2.3)+\left\{p_{0}>2 p_{1}>p_{1}\right\} \Rightarrow p_{0}>2 p_{1}>p_{g}>p_{1}$.

$\Rightarrow\left\{\mathrm{p}_{0}>\mathbf{p}_{\mathrm{g}}>\mathrm{p}_{1}.\right\}$ contradiction. $\because\left(\mathrm{p}_{1}\right.$ and $\mathrm{p}_{0}$ are adjacent prime numbers.)

$\therefore \mathrm{p}_{0} \ngtr 2 \mathrm{p} 1$.

Theorem( $\left.\omega_{1}\right)(Q E D)$.

\subsubsection{2}

Theorem $\left(\omega_{2}\right)$ :

$A_{n}, B_{n}, C_{n}$, If $:\left\{2 n+2=p_{x}+p_{y} \cdot\right.$ set up $\left.\triangle 1: p_{x} \geqslant p_{y}\right\}$

There must be: $\mathrm{p}_{\mathrm{x}}=\mathrm{p}_{0}$

\section{Proof:}

If: $p_{x}+p_{y}=2(n+1)$

$(\mathrm{w})+(2): \quad \mathrm{p}_{0}+\mathbf{3} \geqslant \mathrm{p}_{\mathrm{x}}+\mathrm{p}_{\mathrm{y}}$

【(w): $\mathrm{p}_{0}+1 \geqslant 2 \mathrm{n} \Rightarrow \mathrm{p}_{0}+1+2 \geqslant 2 \mathrm{n}+2=\mathrm{p}_{\mathrm{x}}+\mathrm{p}_{\mathrm{y}} \Rightarrow \mathrm{p}_{0}+3 \geqslant \mathrm{p}_{\mathrm{x}}+\mathrm{p}_{\mathrm{y}} 】$

$\because \quad$ (the smallest prime in $\mathrm{k}$ is $\mathbf{3}$ )

$\therefore \quad p_{\mathbf{y}} \geqslant 3$

$\therefore(3) \Rightarrow \quad p_{0} \geqslant p_{x}$

(4)

$\because\left\{(3.4) \Rightarrow\left\{(2.4)+\left\{3,5,7,11, \ldots, \mathbf{p}_{2}, \mathbf{p}_{1}\right\} \neq \triangleright 2(\mathbf{n}+1)=\mathbf{p}_{\mathbf{i}}+\mathbf{p}_{\mathrm{ii}}\right\}\right.$

$\therefore\left\{(1)+(5) \Rightarrow p_{x} \notin\left\{3,5,7,11, \ldots, p_{2}, p_{1}\right\} \Rightarrow p_{x}>p_{1}\right\}$

【reason: Hypothesis: $\mathrm{p}_{\mathrm{x}} \in\left\{3,5,7,11, \ldots, \mathrm{p}_{2}, \mathrm{p}_{1}\right\}$

$\because p_{x} \geqslant p_{y} \Rightarrow\left\{p_{x}, p_{y}\right\} \in\left\{3,5,7,11, \ldots, p_{2}, p_{1}\right\}$

$\left\{3,5,7,11, \ldots, p_{2}, p_{1}\right\} \Rightarrow \quad p_{x}+p_{y}=2(n+1) \quad$ Contradiction with (5).

$\therefore p_{\times} \notin\left\{3,5,7,11, \ldots, p_{2}, p_{1}\right\} \Rightarrow p_{x}>p_{1} 】$

$\{(4)+(6)\} \Rightarrow \mathrm{p}_{0} \geqslant \mathrm{p}_{\mathrm{x}}>\mathrm{p}_{1}$

Because $\mathrm{p}_{0}>\mathrm{p}_{1}$, and the prime number: $\mathrm{p}_{0}$ and $\mathrm{p}_{1}$ adjacent.

$\left\{\mathrm{p}_{0}\right.$ and $\mathrm{p}_{1}$ adjacent. $\left.+(7)\right\} \Rightarrow \therefore \mathrm{p}_{\mathrm{x}}=\mathrm{p}_{0}$

$\left(\omega_{2}\right)$ (QED). 


\subsubsection{Theorem $\left(\omega_{3}\right)$ :}

Known: $\left\{\mathrm{A}_{\mathrm{n}}, \mathrm{B}_{\mathrm{n}}, \mathrm{C}_{\mathrm{n}},(2.2),(2.4), \mathrm{p}_{1}\right.$ and $\mathrm{p}_{0}$ are adjacent prime numbers , $\mathrm{p}_{0}>\mathrm{p}_{1}$,

$\beta$ line: $2 \rightarrow 4 \rightarrow 6 \rightarrow 8 \rightarrow \ldots \rightarrow 2(n-6) \rightarrow 2(n-5) \rightarrow 2(n-4) \rightarrow 2(n-3) \rightarrow 2(n-2)$

к line: $3 \rightarrow 5 \rightarrow 7 \rightarrow 11 \rightarrow 13 \rightarrow 17 \rightarrow 19 \rightarrow \ldots \quad \rightarrow p_{2} \rightarrow p_{1}$

(2.2) Extreme law : Not allowed: $\left.\mathbf{2 n + 2}=\mathrm{p}_{\mathrm{x}}+\mathrm{p}_{\mathrm{y}}\right\}$ There must be: $\mathbf{p}_{0}-\mathrm{p}_{1}>\mathbf{2}(\mathbf{n}-\mathbf{2})$

\section{Proof:}

$\mathrm{p}_{1}$ and $\mathrm{p}_{0}$ are adjacent prime numbers, $\mathrm{p}_{0}>\mathrm{p}_{1}$,

$\left\{\because\right.$ there is no prime number between $\mathrm{p}_{1}$ and $\mathrm{p}_{0} \quad \therefore \mathrm{p}_{1}$ takes precedence over other prime numbers and approaches $\mathrm{p}_{0}$.

Application

If: $\mathrm{p}_{0}-\mathrm{p}_{\mathrm{x}}=2$, There must be: $\mathrm{p}_{1}=\mathrm{p}_{\mathrm{x}}$

$\because$ there is no odd number between $p_{0}$ and $p_{x}, \because p_{0}>p_{1} \geqslant p_{x}$

If: $\mathrm{p}_{1} \neq \mathrm{p}_{\mathrm{x}}$ There must be: $\mathrm{p}_{0}>\mathrm{p}_{1}>\mathrm{p}_{\mathrm{x}} \rightarrow \mathrm{p}_{1}$ is even $\rightarrow$ contradictory $\therefore \mathrm{p}_{1}=\mathrm{p}_{\mathrm{x}}$

If: $\mathrm{p}_{0}-\mathrm{p}_{\mathrm{x}}=4, \mathrm{p}_{0}-\mathrm{p}_{1} \neq 2$. There must be: $\mathrm{p}_{1}=\mathrm{p}_{\mathrm{x}}$

$\because$ The integer between $\mathrm{p}_{0}$ and $\mathrm{p}_{\mathrm{x}}$ has only an odd number $\mathrm{t} . \quad \because \mathrm{p}_{0}-2 \neq \mathrm{p}_{1} \rightarrow \mathrm{p}_{1} \neq \mathrm{t}$

$\because \mathrm{p}_{0}>\mathrm{p}_{1} \geqslant \mathrm{p}_{\mathrm{x}}, \quad$ If: $\mathrm{p}_{1} \neq \mathrm{p}_{\mathrm{x}} \rightarrow \mathrm{p}_{0}>\mathrm{p}_{1}>\mathrm{p}_{\mathrm{x}} \rightarrow \mathrm{p}_{1}$ is even $\rightarrow$ contradictory. $\therefore \mathrm{p}_{1}=\mathrm{p}_{\mathrm{x}}$

If: $\quad \mathrm{p}_{0}-\mathrm{p}_{\mathrm{x}}=6, \mathrm{p}_{0}-\mathrm{p}_{1} \neq 2, \mathrm{p}_{0}-\mathrm{p}_{1} \neq 4$. There must be: $\mathrm{p}_{1}=\mathrm{p}_{\mathrm{x}}$

$\because$ The integer between $p_{0}$ and $p_{x}$ has only two odd numbers $t, t_{1}$.

$\because\left\{\mathrm{p}_{0}-2 \neq \mathrm{p}_{1}, \mathrm{p}_{0}-\mathrm{p}_{1} \neq 4\right\} \rightarrow \mathrm{p}_{1} \neq\left\{\mathrm{t}, \mathrm{t}_{1}\right\}$

$\because \mathrm{p}_{0}>\mathrm{p}_{1} \geqslant \mathrm{p}_{\mathrm{x}}, \quad$ If: $\mathrm{p}_{1} \neq \mathrm{p}_{\mathrm{x}} \rightarrow \mathrm{p}_{0}>\mathrm{p}_{1}>\mathrm{p}_{\mathrm{x}} \rightarrow \mathrm{p}_{1}$ is even $\rightarrow$ contradictory. $\therefore \mathrm{p}_{1}=\mathrm{p}_{\mathrm{x}}$

The same logic is extended (omitted). $\} \quad$ Record as

(x)

$\because \quad$ Extreme law (2.2): not allowed: $2 \mathbf{n}+\mathbf{2}=\mathrm{p}_{\mathrm{x}}+\mathrm{p}_{\mathrm{y}}$

$\therefore \quad 2 \mathrm{n}+2 \neq \mathrm{p}_{\mathrm{x}}+\mathrm{p}_{\mathrm{y}}$

Under what conditions does $\left(2 \mathrm{n}+\mathbf{2}=\mathrm{p}_{\mathrm{x}}+\mathrm{p}_{\mathrm{y}}\right)$ not exist?

Only if we find the condition that $2 \mathrm{n}+2=\mathrm{p}_{\mathrm{x}}+\mathrm{p}_{\mathrm{y}}$ holds,

To get: $2 \mathrm{n}+2 \neq \mathrm{p}_{\mathrm{x}}+\mathrm{p}_{\mathrm{y}}$

What are the conditions for the establishment of $\left(2 n+2=p_{x}+p_{y}\right)$ ?

If: $2 \mathrm{n}+2=\mathrm{p}_{\mathrm{x}}+\mathrm{p}_{\mathrm{y}}$ holds.

$\left(\omega_{2}\right)+\left(2 \mathrm{n}+2=\mathrm{p}_{\mathrm{x}}+\mathrm{p}_{\mathrm{y}}\right) \Rightarrow 2 \mathrm{n}+2=\mathrm{p}_{0}+\mathrm{p}_{\mathrm{y}}$

Get: $\mathrm{p}_{\mathrm{x}}=\mathrm{p}_{0}$

What is the value of prime $\mathrm{p}_{\mathrm{y}}$ ?

$\because 2 p_{1} \geqslant 2 n \Rightarrow 2 p_{1}+2 \geqslant 2 n+2$ 
$\because \mathbf{p}_{\mathbf{0}}>\mathbf{p}_{\mathbf{1}} \Rightarrow \mathbf{p}_{\mathbf{0}} \geqslant \mathbf{p}_{1}+\mathbf{1} \quad \because$ (odd) $\neq$ (even)

$\therefore p_{0}>p_{1}+1 \Rightarrow 2 p_{0}>2 p_{1}+2$

$\because\left\{\left(2 p_{1}+2 \geqslant 2 n+2\right),\left(2 p_{0}>2 p_{1}+2\right)\right\} \quad \therefore 2 p_{0}>2 n+2$

$\because\left\{\left(2 n+2=p_{0}+p_{y}\right),\left(2 p_{0}>2 n+2\right)\right\} \quad \therefore p_{0}>p_{y}$

$\therefore \mathrm{p}_{\mathrm{y}} \in\left\{3,5,7,11,13,17,19, \ldots, \mathrm{p}_{2}, \mathrm{p}_{1}\right\}$

$\because \quad\left\{3,5,7,11,13,17,19, \ldots, p_{2}, p_{1}\right\}+(2.4) \Rightarrow \mathbf{C}_{\mathbf{n}}$

$\because \quad\{$ Level 2, level 4, level 6, ..., Level 2 (n-3), level 2 (n-2) $\} \in \mathbf{C}_{\mathbf{n}}$

$\therefore \quad\left\{3,5,7,11,13,17,19, \ldots, \mathrm{p}_{2}, \mathrm{p}_{1}\right\} \in\{$ Level 2, level 4, level 6, ..., Level 2 (n-3), level 2 (n-2)\}

$\therefore p_{\mathrm{y}} \in\{$ Level 2, level 4, level 6,... Level $2(\mathrm{n}-3)$, level $2(\mathrm{n}-2)\}$

If: $\mathrm{p}_{\mathrm{y}} \in\{$ Level 2$\}$

$\Rightarrow\left\{\mathrm{p}_{\text {y }} \in \mathbf{p}_{\mathbf{a}}\right\} \Rightarrow\left(\mathrm{p}_{\mathrm{y}}=\mathbf{p}_{\text {ai }}\right) \Rightarrow 2 \mathrm{n}+2=\mathrm{p}_{0}+\mathrm{p}_{\mathrm{ai}}$

$\because$ Level 2: $p_{a i} \quad \therefore$ Level 2: $\left(p_{a i i}+2+p_{a i}=2(n+1)\right) \in\left\{p_{a 1}+2+p_{a 2}=p_{a 3}+2+p_{a 4}=\ldots=2(n+1)\right\}$

$\left\{\left(2 \mathrm{n}+2=\mathrm{p}_{0}+\mathrm{p}_{\mathrm{ai}}\right),\left(\mathbf{p}_{\text {aii }}+2+\mathrm{p}_{\mathrm{ai}}=2(\mathrm{n}+1)\right)\right\} \Rightarrow \mathrm{p}_{\text {aii }}+2=\mathrm{p}_{0}$

$\left(\mathbf{p}_{\text {aii }}+2=\mathrm{p}_{0}\right)+(\mathrm{x}) \Rightarrow \mathbf{p}_{\text {aii }}=\mathbf{p}_{1}$

$\therefore\left(\mathrm{p}_{\text {aii }}+2=\mathrm{p}_{0}\right) \Rightarrow\left(\mathrm{p}_{1}+2=\mathrm{p}_{0}\right) \Rightarrow\left(\mathrm{p}_{0}-\mathrm{p}_{1}=2\right)$

$\therefore\left\{2 \mathrm{n}+2=\mathrm{p}_{\mathrm{x}}+\mathrm{p}_{\mathrm{y}}\right\} \Rightarrow\left(\mathrm{p}_{0}-\mathrm{p}_{1}=2\right) \quad$ Record as $\quad$ (i)

If: Level $2 \quad \mathbf{p a}_{\mathbf{a}}+\mathbf{2}=\mathbf{p}$

$\left(\mathrm{p}_{\mathrm{a}}+\mathbf{2}=\mathbf{p}\right) \Rightarrow\left(\right.$ Level 2) $\Rightarrow \mathbf{p}+\mathbf{p}_{\mathbf{a i}}=\mathbf{2 n + 2} \Rightarrow\left(\mathbf{p}_{\mathbf{x}}+\mathbf{p}_{\mathbf{y}}=\mathbf{2 n}+\mathbf{2}\right)$

$\left(\omega_{2}\right)+\left(p+p_{a i}=2 n+2\right) \Rightarrow p=p_{0}$

$\therefore\left(\mathrm{p}_{\mathrm{a}}+2=\mathrm{p}\right) \Rightarrow \mathrm{p}_{\mathrm{a}}+2=\mathrm{p}_{0}$

$(\mathbf{x})+\left(\mathrm{p}_{\mathrm{a}}+2=\mathrm{p}_{0}\right) \Rightarrow \mathrm{p}_{1}+2=\mathrm{p}_{0}$

$\therefore\left(\mathrm{p}_{\mathrm{a}}+2=\mathrm{p}\right) \cong\left(\mathrm{p}_{1}+2=\mathrm{p}_{0}\right)$

$\therefore\left(p_{a}+2=p\right)=\left(p_{1}+2=p_{0}\right) \Rightarrow\left(p_{x}+p_{y}=2 n+2\right)$ Record as

(i)+(ii) Get: $\quad\left\{\left(\mathrm{p}_{\mathrm{x}}+\mathrm{p}_{\mathrm{y}}=2 \mathrm{n}+2\right) \Leftrightarrow\left(\mathrm{p}_{0}-\mathrm{p}_{1}=2\right)\right\} \quad$ Record as $\quad$ (j)

(j) The formula proves that the condition of $\left(\mathbf{2 n + 2}=\mathrm{p}_{\mathrm{x}}+\mathrm{p}_{\mathrm{y}}\right)$ in Level 2 is: $\mathbf{p}_{0}-\mathrm{p}_{1}=\mathbf{2}$

(2.2 ) Extreme laws do not allow: $2 n+2=p_{x}+p_{y} \Rightarrow 2 n+2 \neq p_{x}+p_{y}$

$\therefore\left\{(\mathrm{j})+\left(2 \mathrm{n}+2 \neq \mathrm{p}_{\mathrm{x}}+\mathrm{p}_{\mathrm{y}}\right)\right\} \Rightarrow \mathrm{p}_{0}-\mathrm{p}_{1} \neq 2$

If: $\mathrm{p}_{\mathrm{y}} \in\{$ Level 4$\}$

$\Rightarrow\left\{p_{y} \in p_{b}\right\} \Rightarrow\left(p_{y}=p_{b i}\right) \Rightarrow 2 n+2=p_{0}+p_{b i}$

$\because$ Level 4: $p_{b i} \quad \therefore$ Level 4: $\left(p_{b i i}+4+p_{b i}=2(n+1)\right) \in\left\{p_{b 1}+4+p_{b 2}=p_{b 3}+4+p_{b 4}=\ldots=2(n+1)\right\}$

$\left\{\left(2 \mathrm{n}+2=\mathrm{p}_{0}+\mathrm{p}_{\mathrm{bi}}\right),\left(\mathrm{p}_{\mathrm{bii}}+4+\mathrm{p}_{\mathrm{bi}}=\mathbf{2}(\mathrm{n}+1)\right)\right\} \Rightarrow \mathrm{p}_{\mathrm{bii}}+4=\mathrm{p}_{0}$

$\left(\mathbf{p}_{\text {bii }}+4=\mathrm{p}_{0}\right)+(\mathrm{x}) \Rightarrow \mathbf{p}_{\text {bii }}=\mathbf{p}_{1}$

$\therefore\left(\mathbf{p}_{\text {bii }}+4=\mathrm{p}_{0}\right) \Rightarrow\left(\mathbf{p}_{1}+4=\mathrm{p}_{0}\right) \Rightarrow\left(\mathrm{p}_{0}-\mathbf{p}_{1}=4\right)$ 
$\therefore\left\{2 \mathrm{n}+2=\mathrm{p}_{\mathrm{x}}+\mathrm{p}_{\text {y }}\right\} \Rightarrow\left(\mathrm{p}_{0}-\mathrm{p}_{1}=2\right)$

If: Level $4 \mathbf{p}_{\mathbf{b}}+\mathbf{+ 4}=\mathbf{p}$,

$\left(\mathbf{p}_{\mathbf{b}}+\mathbf{4}=\mathbf{p}\right) \Rightarrow\left(\right.$ Level 4) $\Rightarrow \mathbf{p}+\mathbf{p}_{\mathbf{b i}}=\mathbf{2 n}+\mathbf{2} \Rightarrow\left(\mathbf{p}_{\mathbf{x}}+\mathbf{p}_{\mathbf{y}}=\mathbf{2 n}+\mathbf{2}\right)$

$\left(\omega_{2}\right)+\left(p+p_{b i}=2 n+2\right) \Rightarrow p=p_{0}$

$\therefore\left(\mathrm{p}_{\mathrm{b}}+4=\mathrm{p}\right) \Rightarrow \mathrm{p}_{\mathrm{b}}+4=\mathrm{p}_{0}$

$(\mathbf{x})+(8)+\left(\mathrm{p}_{\mathrm{b}}+4=\mathrm{p}_{0}\right) \Rightarrow \mathrm{p}_{1}+4=\mathrm{p}_{0}$

$\therefore\left(\mathrm{p}_{\mathrm{b}}+4=\mathrm{p}\right) \cong\left(\mathrm{p}_{1}+4=\mathrm{p}_{0}\right)$

$\therefore\left(\mathrm{p}_{\mathrm{b}}+4=\mathrm{p}\right)=\left(\mathrm{p}_{1}+4=\mathrm{p}_{0}\right) \Rightarrow\left(\mathrm{p}_{\mathrm{x}}+\mathrm{p}_{\mathrm{y}}=2 \mathrm{n}+2\right)$

The same logic leads to: $\left\{\left(\mathrm{p}_{\mathrm{x}}+\mathrm{p}_{\mathrm{y}}=2 \mathrm{n}+2\right) \Leftrightarrow\left(\mathrm{p}_{0}-\mathrm{p}_{1}=4\right)\right\} \quad$ Record as $(\mathrm{jj})$

(jj) The formula proves that the condition of $\left(2 n+2=p_{x}+p_{y}\right)$ in Level 4 is: $p_{0}-p_{1}=4$

(2.2) Extreme laws do not allow: $2 \mathrm{n}+2=\mathrm{p}_{\mathrm{x}}+\mathrm{p}_{\mathrm{y}} \Rightarrow 2 \mathrm{n}+2 \neq \mathrm{p}_{\mathrm{x}}+\mathrm{p}_{\mathrm{y}}$

$\therefore\left\{(\mathrm{jj})+\left(2 \mathrm{n}+2 \neq \mathrm{p}_{\mathrm{x}}+\mathrm{p}_{\mathrm{y}}\right)\right\} \Rightarrow \mathrm{p}_{0}-\mathrm{p}_{1} \neq 4$

If: $\mathrm{p}_{\mathrm{y}} \in\{$ Level 4$\}$

The same logic leads to: $\left\{\left(2 \mathrm{n}+2 \neq \mathrm{p}_{\mathrm{x}}+\mathrm{p}_{\mathrm{y}}\right)\right\} \Rightarrow \mathrm{p}_{0}-\mathrm{p}_{1} \neq \mathbf{6}$

...... Recursive derivation $\rightarrow$

$\beta$ line: $2 \rightarrow 4 \rightarrow 6 \rightarrow 8 \rightarrow \ldots \rightarrow 2(n-6) \rightarrow 2(n-5) \rightarrow 2(n-4) \rightarrow 2(n-3) \rightarrow 2(n-2)$

$\mathbf{C}_{\mathbf{n}}$ : (Level 2 (n-2)) also follows the same principle: $\mathrm{p}_{0}-\mathrm{p}_{1} \neq 2(\mathrm{n}-2)$

$\left\{\mathrm{p}_{0}>\mathrm{p}_{1}\right.$

$\mathrm{p}_{0}-\mathrm{p}_{1} \neq 2$

$\mathrm{p}_{0}-\mathrm{p}_{1} \neq 4$

$\mathrm{p}_{0}-\mathrm{p}_{1} \neq 6$

$\mathrm{p}_{0}-\mathrm{p}_{1} \neq 8$

...

$\mathrm{p}_{0}-\mathrm{p}_{1} \neq 2(\mathrm{n}-3)$

$\left.\mathrm{p}_{0}-\mathrm{p}_{1} \neq 2(\mathrm{n}-2)\right\} \Rightarrow \mathrm{p}_{0}-\mathrm{p}_{1}>2(\mathrm{n}-2)$

$\left(\omega_{3}\right)$ (QED).

【Friendly tip: Goldbach's conjecture ball, kick into each branch of Goldbach's conjecture $\mathbf{C}_{\mathbf{n}}$, and the ball is not allowed to encounter Goldbach's conjecture conclusion in each path. Then we get: $\mathrm{p}_{0}-\mathrm{p}_{1}>2(\mathrm{n}-2)$.】

3.2.1.4 Theorem $\left(\omega_{4}\right): \quad p_{0}-p_{1}>2(n-2) \quad$ violates "Bertrand Chebyshev theorem".

Proof:

$\left(\omega_{3}\right) \Rightarrow p_{0}-p_{1}>2(n-2) \Rightarrow p_{0}-p_{1}>2 n-4 \geqslant p_{1}+p_{i}-4 . 【 \because 2 n \geqslant p_{1}+p_{i} 】$

$\therefore p_{0}>\left(2 p_{1}+p_{i}-4\right) \quad \therefore \quad p_{0} \geqslant\left(2 p_{1}+p_{i}-3\right)$ 
$\because \quad$ (the smallest prime in $k$ is 3$) \Rightarrow p_{i} \geqslant 3 \quad \therefore\left(p_{0} \geqslant 2 p_{1}+p_{i}-3\right) \Rightarrow \quad p_{0} \geqslant 2 p_{1}$

$\because \quad \therefore \mathrm{p}_{0}>2 \mathrm{p}_{1}$

Theorem $\left(\omega_{1}\right)$ Get: $p_{0}>2 p_{1}$ violates the "Bertrand Chebyshev theorem". $\left(\omega_{4}\right)$ (QED).

\subsubsection{Summary: Causes of contradictions}

It is proved that it is wrong to quote "extreme $\operatorname{law}(2.2) "$ in this process.It leads to contradictions.

$\mathrm{A}_{2} \rightarrow \mathrm{A}_{\mathrm{n}}$ can only be a pseudo stop, There can be no true stop.

As long as one of them does not quote the "extreme law(2.2)", You get:

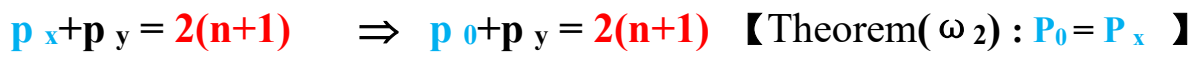

$\left(\omega_{2}\right) \Rightarrow$ New $(\kappa$ line $): 3 \rightarrow 5 \rightarrow 7 \rightarrow 11 \rightarrow 13 \rightarrow 17 \rightarrow 19 \rightarrow \ldots . . . \mathrm{p}_{1} \rightarrow \mathrm{p}_{0}$

At this time, the complete proof $A_{n}$ is followed by $p_{0}+p_{y}=2(n+1)$

(3.2 ) (QED).

(3.1) and (3.2) are equivalent: (3.2) (QED) $\Rightarrow$ (3.1) (QED).

(3.1) and (3.2) are equivalent: (3.2) (QED) $\Rightarrow$ (3.1) (QED).

Complete the mathematical complete induction:

\{A1 ( $\mathrm{K}$ line:3) (3.1) (QED),

An ( $\mathrm{k}$ line:3,5,7,...p 1 ) (3.1) (QED),

$\mathbf{A}_{\mathbf{n}+\mathbf{1}}(\mathrm{k}$ line:3,5,7,...p1, po $)$ (3.1) (QED)\}.

"Authenticity stop" will not appear, so it is always infinite. $\Rightarrow(3.1) \quad$ (QED).

\section{5}

\section{Statement:}

-The author has no relevant financial or non-financial interests to disclose.

-The author has no conflict of interest related to the content of this article.

-All authors warrant that they have no relationship with any organization or entity or are involved in any subject or material of financial or non-financial interest discussed herein.

-The author has no economic or proprietary interest in any of the materials discussed herein.

-Funding (supported by the author) 
-Publication consent (consent)

\section{6}

\section{References cited:}

[1] Background of the study: Euler's version, that is, any even number greater than 2 can be written as the sum of two prime numbers, also known as "strong Goldbach conjecture" or "Goldbach conjecture about even numbers". https://www.springer.com/gp/book/9783034808804

[2] Bertrand Chebyshev theorem: if the integer $\mathbf{n}>\mathbf{3}$, then there is at least one prime $\mathbf{P}$, which conforms to $\mathbf{n}<\mathbf{p}<\mathbf{2 n - 2}$. Another slightly weaker argument is: for all integers $\mathrm{n}$ greater than $\mathbf{1}$, there is at least one prime $\mathrm{P}$, which conforms to $\mathbf{n}<\mathbf{p}<$ 2n.

https://www. researchgate. net/publication/228592091_A Generalization o $\underline{\text { f Erdos's Proof of Bertrand-Chebyshev Theorem }}$

[3] The theorem of infinite number of primes: After each prime, you can always find an adjacent prime.

https://www. researchgate. net/publication/266044680 THE NUMBER OF PRIM ES IS INFINITE 